PROCEEDINGS OF THE

AMERICAN MATHEMATICAL SOCIETY

Volume 126, Number 10, October 1998, Pages 2835-2840

S 0002-9939(98)04396-2

\title{
GLASNER SETS AND POLYNOMIALS IN PRIMES
}

\author{
R. NAIR AND S. L. VELANI
}

(Communicated by William W. Adams)

\begin{abstract}
A set of integers $S$ is said to be Glasner if for every infinite subset $A$ of the torus $\mathbb{T}=\mathbb{R} / \mathbb{Z}$ and $\varepsilon>0$ there exists some $n \in S$ such that the dilation $n A=\{n x: \quad x \in A\}$ intersects every integral of length $\varepsilon$ in $\mathbb{T}$. In this paper we show that if $p_{n}$ denotes the $n$th prime integer and $f$ is any non-constant polynomial mapping the natural numbers to themselves, then $\left(f\left(p_{n}\right)\right)_{n \geq 1}$ is Glasner. The theorem is proved in a quantitative form and generalizes a result of Alon and Peres (1992).
\end{abstract}

\section{INTRODUCTION}

Following D. Berend and Y. Peres $[\mathrm{BP}]$ we say a set $S$ of integers is Glasner if for every infinite set $A$ contained in the one-dimensional torus $\mathbb{T}=\mathbb{R} / \mathbb{Z}$ and $\varepsilon>0$, some dilation $n A=\{n x: \quad x \in A\}$ with $n \in S$ is $\varepsilon$-dense (that is, $n A$ intersects every interval of length $\varepsilon$ ). This definition is motivated by the 1979 result of $\mathrm{S}$. Glasner $[\mathrm{G}]$ in which he showed that given an infinite set $A \subset \mathbb{T}$ there exists a natural number $n$ such that $n A$ is $\varepsilon$-dense in $\mathbb{T}$.

In $[\mathrm{BP}]$ and also $[\mathrm{AP}]$ it is shown that sequences other than the natural numbers are also Glasner. For instance in $[\mathrm{BP}]$ it is shown that if $P$ is a non-constant polynomial with integer coefficients, then $\{P(n): n \in \mathbb{N}\}$ is Glasner, and in [AP] it is shown that $\left\{p_{n}: n \in \mathbb{N}\right\}$, where $p_{n}$ denotes the $n$th rational prime, is also Glasner. In $[\mathrm{AP}]$ too there is a greater emphasis on the quantitative forms of results in $[\mathrm{BP}]$. The methods of $[\mathrm{AP}]$ are Fourier analytic and it is this which allows the more quantitative forms of the result. This means that given $\varepsilon>0$ a lower bound for \#A can be given in terms of $\varepsilon$ for an arbitrary finite set $A$ to ensure that there is some element $n \in S$ such that the dilation $n A$ is $\varepsilon$-dense. In the opposite direction it is known that finite unions of sequences $\left(k_{n}\right)_{n \geq 1}$ such that

$$
\liminf _{n \rightarrow \infty} \frac{k_{n+1}}{k_{n}} \geq g>1
$$

for some $q$ are not Glasner $[\mathrm{BP}]$. In this paper the following theorem is proved

Theorem 1. Let $f$ be a non-constant polynomial of degree $L \geq 1$ mapping the natural numbers to themselves and suppose $\delta>0$. There exists a positive real number $\varepsilon(f, \delta)$ such that if $0<\varepsilon<\varepsilon(f, \delta)$, then any set $X$ contained in $\mathbb{T}$ of

Received by the editors August 19, 1996 and, in revised form, March 3, 1997.

1991 Mathematics Subject Classification. Primary 11K38; Secondary 11K06, 11J13.

(C)1998 American Mathematical Society 
cardinality

$$
s>\left(\frac{1}{\varepsilon}\right)^{2 L+\delta}
$$

has an $\varepsilon$-dense dilation of the form $f(p) X$, for some rational prime integer $p$.

The theorem is easily seen to be a generalization of Theorem 6.3 [AP], in particular it allows us to combine the two separate statements of the theorem in [AP] by considering polynomials in primes.

The paper is organized as follows. In Section 2, we group together various concepts and results required in proving Theorem 1. In Section 3 we give the proof of Theorem 1.

\section{Some PRELIMINARY LEMmas}

In this section we state some technical results required in the sequel. The first is abstracted from special cases dealt with in $[\mathrm{AP}]$.

Lemma 2. Given $\varepsilon>0$, let $X=\left\{x_{1}, \ldots, x_{s}\right\}$ be any set of finitely many points contained in $\mathbb{T}$ such that for every natural number $n$ indexing the sequence $\left(k_{n}\right)_{n \geq 1}$ the dilation $k_{n} X$ is not $\varepsilon$-dense. Then there is an absolute constant $C>0$ such that if

$$
M=\left[\left(\frac{1}{\varepsilon}\right) \log ^{2}\left(\frac{1}{\varepsilon}\right)\right]
$$

we have for any $N>1$

$$
s^{2} \leq\left(\frac{C}{\varepsilon}\right) \sum_{m=1}^{M} \sum_{j=1}^{s} \sum_{l=1}^{s} \frac{1}{N} \sum_{n=1}^{N} e_{m}\left(k_{n}\left(x_{j}-x_{l}\right)\right),
$$

where $e_{m}(t)=\exp (2 \pi i m t)$.

The following result is an immediate consequence of Theorem 4 in [Na].

Lemma 3. If $f$ is a non-constant polynomial mapping the natural numbers to themselves, $\alpha$ is an irrational real number and $p_{n}$ is the nth rational prime number, then the sequence $\left(f\left(p_{n}\right) \alpha\right)_{n \geq 1}$ is uniformly distributed modulo one. In view of Weyl's criterion, this is equivalent to

$$
\lim _{N \rightarrow \infty} \frac{1}{N} \sum_{n=1}^{N} e_{m}\left(f\left(p_{n}\right) \alpha\right)=0,
$$

for every integer $m$ other than zero.

We next need the following classical estimate for rational exponential sums [V].

Lemma 4. Let $\theta$ denote a polynomial of degree $L$ mapping the natural numbers to themselves. Then for any positive $\varepsilon$, there is a constant $C_{1}=C_{1}(\varepsilon, L)>0$ such that

$$
\left|\frac{1}{\phi(b)} \sum_{\substack{a=1 \\(a, b)=1}}^{b} e_{1}\left(\frac{\theta(a)}{b}\right)\right| \leq \frac{C_{1}}{b \frac{1}{L}-\varepsilon},
$$

where $\phi$ denotes Euler's totient function and $(a, b)$ denotes the highest common factor of $a$ and $b$. 
The following is also taken from $[\mathrm{AP}]$.

Lemma 5. Let $\left\{x_{1}, \ldots, x_{s}\right\}$ be an arbitrary set of $s$ distinct points in the unit interval $[0,1)$. For $m \in \mathbb{N}$, denote by $h_{m}$ the number of pairs $(i, j)$ with $1 \leq i<$ $j \leq s$, such that $m\left(x_{i}-x_{j}\right)$ is an integer. Suppose $\beta>0$. Then if $s$ is sufficiently large, for any $m \geq 1$ the partial sum

$$
H_{m}=\sum_{l=1}^{m} h_{l}
$$

satisfies the inequality

$$
H_{m} \leq(s m)^{\beta+1}
$$

In fact, if $m>\exp (10)$, then the estimate can be sharpened to

$$
H_{m} \leq 3(s m)^{(\log \log m)^{-1}+1} .
$$

The trivial upper bound for $H_{m}$ in the above lemma is $s m^{2}$.

\section{Proof of Theorem 1}

Let $\varepsilon>0$ and suppose that $X=\left\{x_{1}, \ldots, x_{s}\right\}$ is a set of $s$ points contained in $\mathbb{T}$ such that the dilation $f\left(p_{n}\right) X$ is not $\varepsilon$-dense for any $n \in \mathbb{N}$. Then on setting

$$
M=\left[\left(\frac{1}{\varepsilon}\right) \log ^{2}\left(\frac{1}{\varepsilon}\right)\right],
$$

Lemma 2 implies that for any $N>1$

$$
s^{2} \leq\left(\frac{C}{\varepsilon}\right) \sum_{m=1}^{M} \sum_{j=1}^{s} \sum_{l=1}^{s} \frac{1}{N} \sum_{n=1}^{N} e_{m}\left(f\left(p_{n}\right)\left(x_{j}-x_{l}\right)\right) .
$$

We now obtain an upper bound for the right hand side of the above expression which in turn will imply the theorem.

As a consequence of Lemma 3 , if for a particular $j$ and $l$ the difference $x_{j}-x_{l}$ is irrational, then the average furthermost to the right in (3.1) tends to zero as $N$ tends to infinity. This means that in estimating the right hand side of (3.1) we need only consider the contribution of the terms in the double sum in $j$ and $l$ for which the corresponding $x_{j}-x_{l}$ is rational.

Note that because $f$ maps the natural numbers to themselves it must have rational coefficients. Hence for any rational $\frac{a}{b}$ in reduced form, we may write

$$
m f(n) \frac{a}{b}=\frac{\left(a_{L}^{\prime} n^{L}+\cdots+a_{1}^{\prime} n\right)}{b^{\prime}}+r=\frac{\theta(n)}{b^{\prime}}+r
$$

where $r$ is a rational, $b^{\prime}$ depends on $b, m$ and the coefficients of $f$, and the highest common factor of the integers $a_{L}^{\prime}, \ldots, a_{1}^{\prime}$ and $b^{\prime}$ is one. As a consequence of Dirichlet's theorem on arithmetic progressions, primes are uniformly distributed among the reduced residue classes modulo $b^{\prime}$. This together with the fact that

$$
\varepsilon_{1}\left(m f\left(p_{n}\right) \frac{a}{b}\right)=e_{1}\left(\frac{\theta(c)}{b^{\prime}}+r\right) \quad \text { whenever } p_{n} \equiv c\left(\bmod b^{\prime}\right)
$$


implies that if $x_{j}-x_{l}=\frac{a}{b}$, then

$$
\lim _{N \rightarrow \infty} \frac{1}{N} \sum_{n=1}^{N} e_{m}\left(f\left(p_{n}\right)\left(x_{j}-x_{l}\right)\right)=\frac{\lambda}{\phi\left(b^{\prime}\right)} \sum_{\substack{a=1 \\\left(a, b^{\prime}\right)=1}}^{b^{\prime}} e_{1}\left(\frac{\theta(a)}{b^{\prime}}\right),
$$

where $\lambda$ has absolute value one.

From Lemma 4 we have that for any positive $\varepsilon_{1}$, there exists a positive constant $C_{1}=C_{1}\left(\varepsilon_{1}, L\right)$ and $C_{1}^{*}=C_{1}^{*}\left(\varepsilon_{1}, f\right)$ such that

$$
\left|\frac{\lambda}{\phi\left(b^{\prime}\right)} \sum_{\substack{a=1 \\\left(a, b^{\prime}\right)=1}}^{b^{\prime}} e_{1}\left(\frac{\theta(a)}{b^{\prime}}\right)\right|<\frac{C_{1}}{\left(b^{\prime}\right)^{\frac{1}{L}-\varepsilon_{1}}} \leq C_{1}^{*}\left(\frac{(m, b)}{b}\right)^{\frac{1}{L}-\varepsilon_{1}} .
$$

Also because there are at most $\frac{M}{r}$ multiplies of $r$ less than $M$ that divide $b$ we know that

$$
\sum_{m=1}^{M}(m, b)^{\frac{1}{L}-\varepsilon_{1}} \leq \sum_{\substack{r \mid b \\ r \leq M}}\left(\frac{M}{r}\right) r^{\frac{1}{L}-\varepsilon_{1}}
$$

We also note that

$$
\sum_{\substack{r \mid b \\ r \leq M}}\left(\frac{M}{r}\right) r^{\frac{1}{L}-\varepsilon_{1}} \leq M \sum_{\substack{r \mid b \\ r \leq M}} 1 \leq M d(b) \leq C_{2} M b^{\varepsilon_{1}},
$$

where $C_{2}$ is a positive constant depending only on $\varepsilon_{1}$ and as usual $d(n)$ denotes the number of integers between one and $n$ inclusive that divide $n$.

It follows from (3.2) and the above estimates that for any distinct $x_{j}, x_{l} \in X$ for which $x_{j}-x_{l}=\frac{a}{b}$ we have the inequality

$$
\sum_{m=1}^{M}\left|\lim _{N \rightarrow \infty} \frac{1}{N} \sum_{n=1}^{N} e_{m}\left(f\left(p_{n}\right)\left(x_{j}-x_{l}\right)\right)\right| \leq C_{3} M b^{-\frac{1}{L}+\varepsilon_{0}},
$$

where $C_{3}=C_{1}^{*} C_{2}$ and $\varepsilon_{0}=2 \varepsilon_{1}$.

Inequality (3.3) is the crucial estimate. To complete the proof we proceed as in [AP] (proof of Theorem 6.3). Let

$$
g_{b}=\#\left\{(j, l): 1 \leq j<l \leq s \text { and } x_{j}-x_{l}=\frac{a}{b} \text { for some } a \text { with }(a, b)=1\right\}
$$

and let

$$
G_{b}=\sum_{i=1}^{b} g_{i}
$$

We can bound the right hand side of (3.1) by the sum over all $1 \leq j, l \leq s$ of the left hand side of (3.3). By partial summation we obtain that for some positive constant 
$C_{4}$ depending only on $\varepsilon_{0}$ and $L$,

$$
\begin{aligned}
s^{2} & \leq C_{4} M \varepsilon^{-1}\left(s+\sum_{b \geq 2} g_{b} b^{-\frac{1}{L}+\varepsilon_{0}}\right) \\
& =C_{4} M \varepsilon^{-1}\left(s+\sum_{b \geq 2} G_{b}\left(b^{-\frac{1}{L}+\varepsilon_{0}}-(b+1)^{-\frac{1}{L}+\varepsilon_{0}}\right)\right) .
\end{aligned}
$$

In order to estimate the sum on the right hand side we use the trivial inequality $G_{b} \leq s^{2}$ when $b>s$ and for $b \leq s$ we make use of Lemma 5 which implies that $G_{b} \leq H_{b} \leq(s b)^{1+\varepsilon_{0}}$, assuming that $s$ is sufficiently large. It follows that for $\varepsilon_{0}$ sufficiently small (depending only on the degree $L$ ),

$$
\begin{gathered}
\sum_{b \geq 2} G_{b}\left(b^{-\frac{1}{L}+\varepsilon_{0}}-(b+1)^{-\frac{1}{L}+\varepsilon_{0}}\right) \\
\leq s^{1+\varepsilon_{0}}\left(\sum_{b=2}^{s} b^{2 \varepsilon_{0}-\frac{1}{L}}+s^{1-\frac{1}{L}}\right) \\
\leq s^{2+3 \varepsilon_{0}-\frac{1}{L}}\left(1+s^{-2 \varepsilon_{0}}\right) .
\end{gathered}
$$

This together with (3.4) implies that

$$
s^{2} \leq C_{5} M \varepsilon^{-1} s^{2+3 \varepsilon_{0}-\frac{1}{L}}
$$

where $C_{5}=C_{5}\left(\varepsilon_{0}, L\right)$ is a positive constant. Finally, on substituting the value of $M$ into the above expression we obtain that for any positive $\delta$ and for any sufficiently small (depending on $\delta$ and $L$ ) positive $\varepsilon$ the inequality

$$
s \leq\left(\frac{1}{\varepsilon}\right)^{2 L+\delta}
$$

is satisfied, under the assumption that $f\left(p_{n}\right) X$ is never $\varepsilon$-dense. This completes the proof of the theorem.

\section{ACKNOWLEDGEMENTS}

We are most grateful to the Sonderforschungsbereich 170 in Göttingen, and to Paddy Patterson in particular, for support and hospitality. We would also like to thank the referee for his/her useful comments which have clarified certain parts of the paper.

\section{REFERENCES}

[AP] N. Alon and Y. Peres, "Uniform dilations", Geometric and Functional Analysis, vol. 2, No. 1 (1992), 1-28. MR 93a:11061

[BP] D. Berend and Y. Peres, "Asymptotically dense dilations of sets on the circle", J. Lond. Math. Soc. (2) 47 (1993), 1-17. MR 94b:11068

[G] S. Glasner, "Almost periodic sets and measures on the Torus", Israel J. Math. 32 (1979), 161-172. MR 80f:54038 
[Na] R. Nair, "On certain solutions of the diophantine equation $x-y=p(z)$ ", Acta. Arith. LXII.1 (1992), 61-71. MR 94a:11124

[V] I. M. Vinogradov, "Selected Works", Springer-Verlag (1985).

Department of Pure Mathematics, University of Liverpool, P.O. Box 147, Liverpool L69 3BX, United Kingdom

E-mail address: nair@liv.ac.uk

Department of Mathematics, Imperial College, University of London, Huxley BuildIng, 180 Queen's Gate, London SW7 2BZ, United Kingdom

E-mail address: s.velani@ic.ac.uk 